Z Epileptol 2018 -31:296-306 https://doi.org/10.1007/s10309-018-0218-6 Online publiziert: 9. November 2018 (c) Springer Medizin Verlag GmbH, ein Teil von Springer Nature 2018

CrossMark

Ingrid E. Scheffer ${ }^{1,2,3,4} \cdot$ Samuel Berkovic ${ }^{1}$ Guiseppe Capovilla ${ }^{5} \cdot$ Mary B. Connolly $^{6}$ Jacqueline French ${ }^{7} \cdot$ Laura Guilhoto $^{8,9} \cdot$ Edouard Hirsch $^{10,11}$. Satish Jain ${ }^{12}$. Gary W. Mathern ${ }^{13}$. Solomon L. Moshé ${ }^{14}$. Douglas R. Nordli ${ }^{15}$. Emilio Perucca ${ }^{16}$. Torbjörn Tomson $^{17} \cdot$ Samuel Wiebe $^{18} \cdot$ Yue-Hua Zhang ${ }^{19} \cdot$ Sameer M. Zuberi ${ }^{20,21}$

' Department of Medicine, The University of Melbourne, Austin Health, Melbourne, Australien; ${ }^{2}$ Department of Paediatrics, Royal Children's Hospital, The University of Melbourne, Melbourne, Australien; ${ }^{3}$ Florey Institute, Melbourne, Australien; ${ }^{4}$ Epilepsy Research Centre, Heidelberg, Australien; ${ }^{5}$ Child Neuropsychiatry Department, Epilepsy Center, C. Poma Hospital, Mantova, Italien; ${ }^{6}$ Department of Pediatrics, British Columbia's Children's Hospital, University of British Columbia, Vancouver, Kanada;

${ }^{7}$ Department of Neurology, NYU School of Medicine, New York, USA; ${ }^{8}$ Department of Neurology, Federal

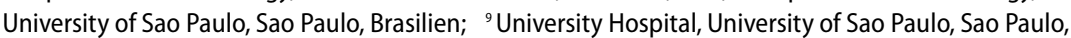
Brasilien; ${ }^{10}$ University Hospital INSERM U 964, Strasbourg, Frankreich; ${ }^{11}$ IDEE, Lyon, Frankreich; ${ }^{12}$ Indian Epilepsy Centre, New Delhi, Indien; ${ }^{13}$ Departments of Neurosurgery, Psychiatry and Biobehavioral Medicine, David Geffen School of Medicine, UCLA, Los Angeles, USA; ${ }^{14}$ Saul R. Korey Department of Neurology, Dominick P. Purpura Department of Neuroscience and Department of Pediatrics, Albert Einstein College of Medicine and Montefiore Medical Center, Bronx, USA; ${ }^{15}$ Division of Neurology, Children's Hospital Los Angeles, Los Angeles, USA; ${ }^{16} \mathrm{C}$. Mondino National Neurological Institute and Clinical Pharmacology Unit, University of Pavia, Pavia, Italien; ${ }^{17}$ Department of Clinical Neuroscience, Karolinska Institute, Stockholm, Schweden; ${ }^{18}$ Departments of Clinical Neurosciences and Community Health Sciences, University of Calgary, Calgary, Kanada; ${ }^{19}$ Department of Pediatrics, Peking University First Hospital, Beijing, Volksrepublik China; ${ }^{20}$ Paediatric Neurosciences Research Group, Fraser of Allander Neurosciences Unit, Royal Hospital for Children, Glasgow, Großbritannien; ${ }^{21}$ School of Medicine, University of Glasgow, Glasgow, Großbritannien

\title{
ILAE-Klassifikation der Epilepsien: Positionspapier der ILAE-Kommission für Klassifikation und Terminologie
}

Nahezu seit ihrer Gründung 1909 hat die „International League Against Epilepsy" (ILAE) kontinuierlich Schritte unternommen, die Klassifikation von Epilepsien $\mathrm{zu}$ verbessern und zu verfeinern. Dabei brachten die von Henri Gastaut in den 1960er-Jahren vorgeschlagenen neuen Konzepte zur Klassifizierung [1-3] diese Bemühungen besonders voran. Intensive Debatten und neue Erkenntnisse führten über die nächsten 2 Dekaden dann $1985 \mathrm{zu}$ der wegweisenden „ILAE Klassifizierung von Epilepsien und epileptischen Syndromen“ "4], die 1989 von einer überarbeiteten und von der ILAEGeneralversammlung ratifizierten Ver-

Dies ist die autorisierte deutschsprachige Übersetzung der Publikation ILAE classification of the epilepsies: Position paper of the ILAE Commission for Classification and Terminology. Epilepsia. 2017, 58(4):512-521. https://doi.org/10.1111/ epi.13709. sion abgelöst wurde [5]. Die Klassifikation von 1989 hatte weltweit großen Einfluss und große Auswirkungen auf die Behandlung und Erforschung von Epilepsien. Die darin enthaltene Arbeit ist durch Anstrengungen vieler über den Verlauf eines Jahrhunderts entstanden; wir sind uns dieser wegweisenden Beiträge zur Entwicklung der Klassifikation der Epilepsien wohl bewusst.

Obwohl viele der in der 1989er-ILAEKlassifikation ausgearbeiteten Konzepte heute noch gültig sind, wurde zuletzt doch immer deutlicher, dass eine Überarbeitung notwendig ist, um dem wissenschaftlichen Erkenntnisgewinn der letzten Jahrzehnte, der unser Verständnisvon Epilepsien sowie unsere Art der Diagnostik und Behandlung von Menschen mit Epilepsie grundlegend verändert hat, Rechnung zu tragen.
Die Klassifikation von Epilepsien ist das wichtigste klinische Handwerkszeug bei der Evaluation eines Patienten, der unter Anfällen leidet. Sie beeinflusst jede klinische Einschätzung. Doch geht ihre Tragweite deutlich über den klinischen Kontext hinaus. Die Klassifikation ergibt den Rahmen für klinische und grundlagenorientierte epileptologische

Ad hoc Task Force der DGfE, ÖGfE und der Schweizerischen Epilepsie-Liga (alphabetisch)

- Prof. Dr. Hajo Hamer, MHBA, Erlangen Deutschland

- Dr. med. Günter Krämer, Zürich, Schweiz

- Prof. Dr. med. Stephan Rüegg, Basel, Schweiz

- Prof. Dr. med. Andreas Schulze-Bonhage, Freiburg, Deutschland

- Univ. Prof. Dr. Mag. Eugen Trinka, FRCP, Salzburg, Österreich 


\section{Kernpunkte}

- Die ILAE präsentiert ein überarbeitetes Grundgerüst der Klassifikation von Epilepsien, ergänzt durch die Klassifikation der Anfallsformen.

- Stufen der Diagnose: Anfallsform, Art der Epilepsie (fokal, generalisiert, kombiniert generalisiert und fokal, unklassifiziert) sowie Epilepsiesyndrom.

- Eine ätiologische Einordnung sollte von der ersten Untersuchung an angestrebt werden sowie bei jedem weiteren Schritt im Rahmen der Diagnostik. Die Epilepsie des jeweiligen Patienten kann mehr als einer ätiologischen Kategorie zugeordnet werden.

- Der Ausdruck "benigne“ wird durch die Begriffe "selbstlimitiert" und "pharmakoresponsiv" ersetzt, um an geeigneter Stelle benützt zu werden.

- Die Bezeichnung „entwicklungsbedingte und epileptische Enzephalopathie" kann je nach Bedarf teilweise oder zur Gänze verwendet werden.

Forschung sowie zur Entwicklung neuer Therapien vor. Sie hat mannigfaltige Anwendungen, beispielsweise als Gerüst für das Verständnis der Anfälle eines Patienten, oder um festzustellen, welche anderen Anfallsarten mit höherer Wahrscheinlichkeit in dem individuellen Fall auftreten könnten, um potenzielle Provokationsfaktoren der Anfälle zu identifizieren, und häufig auch zur Abschätzung der Prognose. Die Klassifikation beinhaltet auch Information über die Risiken möglicher Begleiterkrankungen einschließlich Lernbehinderung und anderer kognitiver Einschränkungen, psychiatrischer Störungen wie der Autismus-Spektrum-Störung sowie über das Sterblichkeitsrisiko, wie beispielsweise „sudden unexpected death in epilepsy“ (SUDEP). Auch beeinflusst die Klassifikation häufig die Wahl antiepileptischer Therapien.

Seit ihrer Einführung in den 1960erJahren hat sich die Klassifikation von Epilepsien stark verändert [6-9]. Die vielen Überarbeitungen spiegeln das fortschreitende Verständnis phänotypischer Muster und grundlegender Mechanismen wider, das weltweit durch wichtige Beiträgen aus klinischer und Grundlagenforschung vorangetrieben wurde. Dieser Gewinn an Wissen ist an vielen Stellen in die Patientenver- sorgung eingeflossen und führt mit der Zeit $\mathrm{zu}$ neuen Behandlungsoptionen, seien es pharmakologische oder diätetische Therapien, epilepsiechirurgische Eingriffe oder die Entwicklung von medizinischen Geräten. Klassifizierung ist somit immer ein dynamischer Prozess, der iterativ auf neue Forschungsergebnisse oder anwachsendes Wissen über diese heterogene Krankheitsgruppe reagiert. Die anhaltende Weiterentwicklung der Klassifikation auch in der Zukunft verspricht weitere Fortschritte in der Versorgung von Patienten.

Klassifizierung führt häufig zu leidenschaftlichen Debatten. Einerseits weil sie auf komplexen, klinischen Konstrukten beruht, die den Epilepsiediagnosen zugrunde liegen, andererseits weil sie in der alltäglichen Praxis eine so zentrale Rolle einnimmt. Die Klassifikation basiert auf dem Fachwissen von Epileptologen und anderen Experten aus der ganzen Welt. Obwohl zweifelsohne das angestrebte Ziel eine wissenschaftlich fundierte Klassifikation ist, reicht unser derzeitiges Verständnis für eine Klassifikation nach streng wissenschaftlichen Maßstäben nicht aus [9]. Dementsprechend basieren die aktuellen Vorschläge auf der Kombination aus neuesten wissenschaftlichen Erkenntnissen und hochkarätigen Expertenmeinungen sowie aufintensiven Beratungen mit Epilepsiefachkräften und der weltweiten Epilepsiegemeinschaft.

Die Diagnostik bei einem Patienten mit epileptischen Anfällen durchläuft mehrere Schritte. Die Anwendung der Anfallsklassifikation setzt voraus, dass der behandelnde Arzt das paroxysmale Ereignis tatsächlich als epileptischen Anfall wertet. Denn es kommt grundsätzlich eine Vielzahl von Differenzialdiagnosen in Betracht, unter anderem konvulsive Synkopen, Parasomnien, Bewegungsstörungen, und andere nichtepileptische Ereignisse (https://www.epilepsydiagnosis. org/epilepsy-imitators.html). Die erfolgte Anfallsklassifizierung ist Voraussetzung für die Epilepsieklassifizierung.

Bei der Klassifikation einer Epilepsie beginnt der behandelnde Arzt mit der Klassifizierung der Anfallsform. Dies ist das Thema des Begleitartikels zur neuen Klassifikation von Anfallsformen [10]. Danach wird die Art der Epilepsie des
Patienten klassifiziert, und in vielen Fällen kann dann die Diagnose eines spezifischen Epilepsiesyndroms gestellt werden. Genauso wichtig ist es, die Ätiologie der Epilepsie des Patienten zu identifizieren. Überlegungen hinsichtlich der Ätiologie sollten bei jedem Schritt im diagnostischen Verlauf angestellt werden. Die Klassifikation der Anfallsform und der Art der Epilepsie berücksichtigen beide die Ergebnisse von Zusatzdiagnostik wie EEG und zerebraler Bildgebung genauso wie die weitergehenden Untersuchungen zur Klärung der Ätiologie der Epilepsie.

Im Folgenden beschreiben wir die erste größere Klassifikation von Epilepsien seit der letzten ratifizierten ILAE-Klassifizierung 1989.

\section{Methoden}

In der Vergangenheit wurden Positionspapiere der ILAE zu grundlegenden Aspekten wie Terminologie, Definition und Klassifikation von Anfällen und Epilepsien immer in der Generalversammlung der ILAE durch Abstimmung der Repräsentanten der verschiedenen weltweiten Fachgesellschaften für Epileptologie ratifiziert [5]. Dieses Vorgehen ist heutzutage nicht mehr optimal, da eine angemessene Einbindung der erheblich gewachsenen globalen Gemeinschaft von Epilepsieexperten auf diese Weise nicht möglich ist. Es würden so auch die Möglichkeiten der modernen Kommunikationstechnologie ungenutzt bleiben. Deshalb hat die ILAE 2013 ein neues Verfahren für die Verabschiedung und Abstimmung von Positionspapieren eingeführt. Dieses Verfahren kommt nur für die Dokumente zur Anwendung, die die offizielle ILAE Position zu Themen widerspiegeln, die dadurch mit der Einigung auf eine gemeinsame Sprache oder gemeinsame Definitionen (z. B. die Definition von Epilepsie; Klassifikation) verbunden sind [11].

Dieses Verfahren ist hochgradig iterativ. Nach der initialen Erstellung des ersten Entwurfs von einer Gruppe von durch die ILAE bestimmten Experten wird das Dokument auf der ILAE-Website veröffentlicht, und Interessenvertreter werden aufgefordert, Kommentare und Kritik einzureichen. Ein weiteres Gremium von 
Z Epileptol 2018 · 31:296-306 https://doi.org/10.1007/s10309-018-0218-6

(c) Springer Medizin Verlag GmbH, ein Teil von Springer Nature 2018

I. E. Scheffer · S. Berkovic · G. Capovilla · M. B. Connolly · J. French · L. Guilhoto · E. Hirsch · S. Jain · G. W. Mathern · S. L. Moshé · D. R. Nordli • E. Perucca $\cdot$ T. Tomson · S. Wiebe · Y.-H. Zhang · S. M. Zuberi

\section{ILAE-Klassifikation der Epilepsien: Positionspapier der ILAE-Kommission für Klassifikation und Terminologie}

\section{Zusammenfassung}

Seit der letzten ratifizierten Epilepsieklassifikation von 1989 führten weitreichende wissenschaftliche Fortschritte zu einem erheblichen Erkenntnisgewinn im Hinblick auf Epilepsien und deren grundlegenden Mechanismen, sodass nun die „Internationale Liga gegen Epilepsie" (ILAE) die Epilepsieklassifikation aktualisiert hat. Als ein grundlegendes Werkzeug für den behandelnden Arzt muss eine Epilepsieklassifikation dynamisch und relevant auf veränderte Konzepte reagieren können, aber gleichzeitig auch verlässlich überall auf der Welt anwendbar bleiben. Die Klassifikation dient v. a. der klinischen Diagnose, ist aber auch für die Forschung, die Entwicklung antiepileptischer Therapien und für die globale Kommunikation wichtig. Die neue Klassifikation basiert auf einem ersten Dokument, das 2013 zur öffentlichen Diskussion gestellt wurde und nach vielen Rückmeldungen der internationalen Epilepsiegemeinschaft dann in mehreren Beratungsrunden angepasst wurde. Sie besteht aus 3 Stufen. Zunächst wird die Anfallsform bestimmt. Dabei wird vorausgesetzt, dass die epileptischen Anfälle des Patienten nach der neuen 2017er-ILAEAnfallsklassifikation eingeordnet wurden. Nach Feststellung der Anfallsform folgt als nächster Schritt die Diagnose der Art der Epilepsie einschließlich fokaler Epilepsien, generalisierter Epilepsien, „kombinierter generalisierter und fokaler Epilepsien" sowie einer Gruppe unklassifizierter Epilepsien. Auf der dritten Stufe wird die Erkrankung einem spezifischen Epilepsiesyndrom zugeordnet. In der neuen Klassifikation wird auf jeder Stufe die Ätiologie mit einbezogen. Dies ist notwendig, da die Ätiologie bei jedem Schritt Konsequenzen für die weitere Behandlung haben kann. Die verschiedenen Ätiologien können in 6 Gruppen eingeordnet werden, die in Bezug auf eine mögliche therapeutische Relevanz definiert wurden. Neue Begriffe werden eingeführt, wie z. B. „entwicklungsbedingte und epileptische Enzephalopathie". Der Ausdruck „benigne“ wird durch die Begriffe "selbstlimitiert" und "pharmakoresponsiv" ersetzt. Es besteht die Hoffnung, dass dieses neue Grundgerüst dabei hilft, die klinische Versorgung der Patienten und die Erforschung der Epilepsien im 21. Jahrhundert zu verbessern.

Schlüsselwörter

Klassifikation - Epilepsie - Epilepsiesyndrome . Terminologie $\cdot$ Ätiologie

\section{ILAE classification of the epilepsies: position paper of the ILAE Commission for Classification and Terminology}

\section{Abstract}

The International League Against Epilepsy (ILAE) classification of the epilepsies has been updated to reflect the gain in understanding of the epilepsies and their underlying mechanisms following the major scientific advances that have taken place since the last ratified classification in 1989. As a critical tool for the practicing clinician, epilepsy classification must be relevant and dynamic to changes in thinking, yet robust and translatable to all areas of the globe. Its primary purpose is for the clinical diagnosis of patients but it is also critical for epilepsy research, development of antiepileptic treatment and communication around the world. The new classification is based on a draft document submitted for public comments in 2013, which was revised to incorporate extensive feedback from the international epilepsy community over several rounds of consultation. It consists of three levels starting with seizure type, where it is assumed that the epileptic seizures of the patient are defined by the new 2017 ILAE seizure classification. After diagnosis of the seizure type, the next step is the diagnosis of the epilepsy type, which includes focal epilepsy, generalized epilepsy, combined generalized and focal epilepsy and also an unclassified epilepsy group. At the third level the disease is assigned to a specific epilepsy syndrome. The new classification incorporates etiology at each stage, emphasizing the need to consider etiology at each step of the diagnosis, as it often carries significant treatment implications. The various etiologies can be assigned to six subgroups, defined with respect to the potential therapeutic consequences. New terminology is introduced, such as developmental and epileptic encephalopathy. The term benign is replaced by the terms selflimiting and pharmacoresponsive, to be used where appropriate. It is hoped that this new framework will assist in improving epilepsy care and research in the twenty-first century.

\section{Keywords}

Classification - Epilepsy - Epilepsy syndromes . Etiology $\cdot$ Terminology
Experten wird ernannt, um die Kommentare und Kritikpunkte zu überprüfen und einzuarbeiten. Dieses Verfahren wird parallel zur einer Peer-review-Begutachtung durch die Zeitschrift, bei der das Dokument zur Publikation eingereicht wird, durchlaufen (http://www. ilae.org/Visitors/Documents/GuidelinePublPolicy-2013Aug.pdf).
Im Falle der vorliegenden überarbeiteten Klassifikation wurde 2010 bereits vor dem Beginn des beschriebenen Verfahrens ein erster Vorschlag von der ILAE-Kommission für Klassifikation und Terminologie veröffentlicht [9]. Der Schwerpunkt lag dabei auf einer transparenten und nachvollziehbaren Terminologie. Die 2010er-Publikation führte zu einer breit gefächerten Diskussion und vielen Kommentaren [12-29]. Darauf folgend wurde eine neue Kommission für Klassifikation und Terminologie vom ILAE Vorstand beauftragt, eine überarbeitete Klassifikation unter Anwendung des beschriebenen Verfahrens für ILAE-Positionspapiere aufzustellen. Die Kommission reichte das ursprüngli- 
che Dokument 2013 ein. Es wurde online gestellt und zur Diskussion freigegeben (s. Scheffer et al. [30]). Lebhafte Debatten und Beiträge folgten mit 128 eingereichten Kommentaren aus 43 Ländern. Die Reaktionen waren so umfassend und das Feedback zu wichtigen Themen so kontrovers, dass das mit der Durchsicht der öffentlichen Kommentare beauftragte Gremium festlegte, dass auch zukünftig öffentliches Mitwirken unabdingbar sein wird, um einen höchstmöglichen Grad an Konsens zu erreichen. Die Strategie des Gremiums, weitere Mitarbeit zu erbitten sowie dem Feedback der verschiedenen Interessenvertreter $\mathrm{zu}$ antworten, ist in einem in Epilepsia Open 2016 erschienenen Artikel beschrieben. In diesem Artikel wurde wiederum die weltweite Epilepsiegemeinschaft $\mathrm{zu}$ erneuter Kommentierung eingeladen [30]. Weitere Rückmeldungen und Meinungen folgten, wurden diskutiert und abgewogen. Das daraus sich ergebende aktuelle Positionspapier stellt die Klassifikation von Epilepsien in 2017 dar.

\section{Klassifikation der Epilepsien}

Die neue Klassifikation der Epilepsien ist eine mehrstufige Klassifikation, die entworfen wurde, um Epilepsien in unterschiedlichen klinischen Kontexten einheitlich zu klassifizieren (• Abb. 1). Dies berücksichtigt die global unterschiedlichen Ressourcen in einem variierenden klinischen Umfeld. So können verschiedene Stufen der Klassifikation erreicht werden, abhängig von den Ressourcen, die dem behandelnden Arzt zur Verfügung stehen. Wo auch immer möglich, sollte eine Diagnose auf allen 3 Stufen angestrebt und die individuelle Ätiologie der Epilepsie im Einzelfall geklärt werden.

\section{Anfallsform}

Der Ausgangspunkt dieser Epilepsieklassifikation ist die Klassifikation der Anfallsformen. Es wird davon ausgegangen, dass der behandelnde Arzt die Diagnose eines epileptischen Anfalls bereits gestellt hat. Die Epilepsieklassifikation ist nicht als diagnostischer Algorithmus gedacht, um epileptische von nichtepileptischen
Ereignissen zu unterscheiden. Die Klassifikation der Anfallsformen ist mit neuer Nomenklatur im Begleitpaper niedergelegt [10]. Anfälle werden klassifiziert als Anfälle mit fokalem, mit generalisiertem oder mit unbekanntem Beginn. In manchen Situationen kann die Klassifizierung nach Anfallsform die bestmögliche Stufe sein, da es u. U. keine Möglichkeit gibt, ein EEG abzuleiten, ein Video aufzuzeichnen oder Bildgebung durchzuführen. In anderen Fällen kann es einfach zu wenig verfügbare Informationen geben, um weitergehende Diagnosen auf höheren Stufen zu stellen, beispielsweise wenn ein Patient nur einen einzelnen Anfall hatte.

\section{Art der Epilepsie}

Die zweite Stufe ist die der Art der Epilepsie und setzt voraus, dass bei dem $\mathrm{Pa}$ tienten eine Epilepsie gemäß der Definition von 2014 diagnostiziert wurde [31]. Die Stufe der Epilepsieart beinhaltet eine neue Kategorie, nämlich „kombinierte generalisierte und fokale Epilepsie“, zusätzlich zu den etablierten Kategorien "generalisierte Epilepsie“ und „fokale Epilepsie“. Sie beinhaltet ebenfalls eine Kategorie „unklassifiziert“. Viele Epilepsien gehen mit mehreren Arten von Anfällen einher.

Bei der Diagnose einer generalisierten Epilepsie treten typischerweise generalisierte Spike-wave-Komplexe im EEG auf. Patienten mit generalisierter Epilepsie können verschiedene Anfallsformen haben wie Absencen, myoklonische, atonische, tonische und tonisch-klonische Anfälle.

Die Diagnose einer generalisierten Epilepsie wird anhand des klinischen Bildes gestellt und von typischen interiktualen Potenzialen im EEG gestützt. Bei einem Patienten mit generalisierten tonisch-klonischen Anfällen und normalem EEG ist Vorsicht geboten. In diesen Fällen sind weitere Hinweise, wie z. B. Myoklonien oder eine passende Familienanamnese, erforderlich, um eine generalisierte Epilepsie diagnostizieren zu können.

Fokale Epilepsien umfassen unifokale und multifokale Epilepsien sowie Anfälle, die eine gesamte Gehirnhälfte einbe- ziehen. Zu der Reihe der möglichen Anfallsformen gehören $u$. a. bewusst erlebter fokaler Anfall, nicht bewusst erlebter fokaler Anfall, fokaler nichtmotorischer Anfall und fokaler mit Übergang zu bilateral tonisch-klonischem Anfall. Das interiktuale EEG zeigt typischerweise fokale epilepsietypische Potenziale. Die Diagnose wird anhand klinischer Befunde gestellt und von den EEG-Befunden gestützt.

Die neue Gruppe der „kombinierten generalisierten und fokalen Epilepsien“ ermöglicht die Klassifikation von Patienten, die sowohl generalisierte als auch fokale Anfälle erleiden. Die Diagnose wird klinisch gestellt und von EEG-Befunden gestützt. Iktuale EEG-Aufzeichnungen sind hilfreich, aber nicht zwingend erforderlich. Das interiktuale EEG kann sowohl generalisierte Spike-waveKomplexe als auch fokale epilepsietypische Potenziale beinhalten. Epilepsietypische Potenziale sind für die Diagnose jedoch nicht zwingend erforderlich. Typische Beispiele für Epilepsiearten, in denen beide Anfallsformen auftreten können, sind u. a. das Dravet-Syndrom und das Lennox-Gastaut-Syndrom.

Die Art der Epilepsie kann die letztmögliche Diagnosestufe sein, falls der behandelnde Arzt sich nicht in der Lage sieht, ein spezifisches Epilepsiesyndrom zu diagnostizieren. Beispiele können sein: Die häufige Situation eines Kindes oder Erwachsenen, welche an einer nichtläsionellen Temporallappenepilepsie leiden, die dann als fokale Epilepsie mit unbekannter Ätiologie einzustufen ist; ein 5-jähriges Kind erleidet generalisierte tonisch-klonische Anfälle mit generalisierten Spike-wave-Komplexen im EEG, wodurch zwar nicht ein bekanntes Epilepsiesyndrom klassifiziert, jedoch nichtsdestotrotz die eindeutige Diagnose einer generalisierten Epilepsie gestellt werden kann. Vorstellbar ist auch das eher seltene Szenario einer 20-jährigen Frau mit sowohl nicht bewusst erlebten fokalen Anfällen als auch Absencen, deren EEG fokale epilepsietypische Potenziale einerseits und generalisierte Spike-waveAktivität andererseits aufweist und in deren MRT keine relevanten Auffälligkeiten nachweisbar sind. Hier liegt die Diagnose 
einer kombinierten generalisierten und fokalen Epilepsie vor.

Der Begriff „unklassifiziert“ sollte denjenigen Fälle vorbehalten bleiben, bei denen klinisch eine Epilepsie besteht, aber nicht bestimmt werden kann, ob diese fokal oder generalisiert ist, weil nicht genügend Informationen zur Verfügung stehen. Dies kann eine Vielzahl von Gründen haben. Es könnte sein, dass es keine Möglichkeit gibt, ein EEG abzuleiten, oder dass die EEG-Befunde nicht weiterführend, z.B. normal, sind. Wenn die Anfallsformen unbekannt sind, kann die Art der Epilepsie aus ähnlichen Gründen unklassifiziert bleiben, obwohl diese beiden nicht konkordant sein müssen. Der Patient könnte beispielsweise mehrere symmetrische tonisch-klonische Anfälle ohne fokale Symptome und mit normalem EEG haben. Dementsprechend ist der Beginn der Anfälle unbekannt, und die Person hat eine unbekannte Epilepsieart.

\section{Epilepsiesyndrom}

Die dritte Stufe ist die Diagnose des Epilepsiesyndroms. Ein Epilepsiesyndrom bezeichnet ein Cluster von Merkmalen inklusive der Anfallsformen, des EEGs und der Befunden der Bildgebung, die häufig in Kombination auftreten. Häufig hängt die Diagnose mit altersabhängigen Faktoren zusammen wie dem Alter bei Beginn und Remission (wo zutreffend), mit anfallsauslösenden Faktoren, tageszeitlicher Variation und manchmal auch mit der Prognose $[4,5]$. Sie kann auch bestimmte Komorbiditäten umfassen wie kognitive und psychiatrische Störungen im Zusammenhang mit bestimmten Befunden im EEG und in der Bildgebung. Sie kann Auswirkungen auf die Ätiologie, Prognose und Behandlung haben. Es ist wichtig, sich zu vergegenwärtigen, dass die Diagnose eines Epilepsiesyndroms nicht notwendigerweise mit einer 1:1Korrelation zu einer bestimmten ätiologischen Diagnose einhergeht und dass sie ein unterschiedliches Ziel verfolgen kann, wie z. B. das Patientenmanagement $\mathrm{zu}$ steuern. Es gibt viele allgemein anerkannte Epilepsiesyndrome, wie z.B. die juvenile Absenceepilepsie, das West-Syndrom und das Dravet-Syndrom. Dabei muss allerdings berücksichtigt werden, dass es keine formelle Klassifikation von Syndromen der ILAE gibt [9]. Die kürzlich entwickelte Fortbildungswebseite der ILAE (epilepsydagnosis.org) ist ein hervorragender Ort, um die verschiedenen Aspekte der Diagnosen zu verstehen und Videos von verschiedenen Anfallsformen sowie EEG-Befunden mehrerer bekannter Syndrome einzusehen.

\section{Idiopathische generalisierte Epilepsien}

In der Gruppe der generalisierten Epilepsien befindet sich die allgemein bekannte und geläufige Untergruppe der idiopathischen generalisierten Epilepsien (IGE). IGE umfassen 4 gut etablierte Epilepsiesyndrome: Absenceepilepsie des Kindesalters, juvenile Absenceepilepsie, juvenile myoklonische Epilepsie und Epilepsie mit ausschließlich generalisierten tonisch-klonischen Anfällen (früher bezeichnet als Aufwach-Grand-Mal, was geändert wurde, weil bei diesem Syndrom ein Anfall zu jeder Tageszeit auftreten kann). Es wurde vorgeschlagen, den Begriff ,idiopathisch“ aus der Nomenklatur der Epilepsieklassifikation zu streichen, da deren Definition lautet: „keine bekannte oder vermutete Ätiologie außer einer möglichen hereditären Prädisposition“ [4]. Der griechische Begriff „,idios“ trägt die Bedeutung von ,,selbst“; , ,eigen“; „persönlich“ und soll dadurch die mögliche genetische Genese widerspiegeln, ohne diese explizit zu nennen. „Idiopathisch" könnte daher als unpräziser Begriff betrachtet werden, insbesondere vor dem Hintergrund der Fortschritte bei der Identifizierung von Genen, die bei vielen Epilepsien eine Rolle spielen, inklusive jenen mit monogener (mit vererbten oder de novo pathogenen Varianten) oder komplexer (polygener, mit oder ohne umweltbedingten Faktoren) Vererbung. Allerdings kann das Wort „genetisch" fälschlicherweise als gleichbedeutend mit "vererbt“ interpretiert werden.

Es erscheint insgesamt treffender, diese Gruppe von Syndromen als ,genetische generalisierte Epilepsien" (GGE) $\mathrm{zu}$ bezeichnen, wenn der behandelnde Arzt der Meinung ist, dass ausreichende Hinweise für diese Klassifikation vorhanden sind. Solche Hinweise stammen aus aufwendiger klinischer Forschung mittels Zwillings- und Familienstudien zur Erblichkeit der betreffenden Syndrome und bedeuten nicht zwingend, dass spezifische genetische Mutationen identifiziert worden sind. Es ist derzeit nur sehr selten der Fall, dass genetische Mutationen als Ursache der Epilepsie eines Patienten nachgewiesen worden sind. Mögliche Ausnahmen sind die frühkindlichen „entwicklungsbedingten und epileptischen Enzephalopathien", bei denen viele Patienten eine pathogene De-novoMutation aufweisen [32].

Nichtdestotrotz bestand weiter der große Wunsch, den Begriff IGE beizubehalten. Die Kommission hat sich deswegen entschieden, dass der Begriff IGE spezifisch für die folgenden 4 epileptischen Syndrome weiterverwendet werden kann: Absenceepilepsie des Kindesalters, juvenile Absenceepilepsie, juvenile myoklonische Epilepsie und Epilepsie mit ausschließlich generalisierten tonisch-klonischen Anfällen. In Einzelfällen kann der Begriff „genetische generalisierte Epilepsie" verwendet werden, wenn der behandelnde Arzt bewusst auf eine mögliche genetische Ätiologie hinweisen möchte.

\section{Selbstlimitierte fokale Epilepsien}

Es existieren mehrere selbstlimitierte, fokale Epilepsien, die typischerweise in der Kindheit beginnen. Am häufigsten ist die selbstlimitierte Epilepsie mit zentrotemporalen „spikes“, früher als „,benigne Epilepsie mit zentrotemporalen spikes" bezeichnet. Andere Epilepsien dieser breit gefassten Gruppe sind die selbstlimitierte Okzipitallappenepilepsie der Kindheit mit einer von Panayiotopoulos beschriebenen früh einsetzenden Form und einer von Gastaut beschriebenen spät einsetzenden Form [33]. Selbstlimitierte Frontallappen- [34], Temporallappen- [35] und Parietallappen- [36] Epilepsien wurden beschrieben, wovon einige in der Adoleszenz oder sogar im erwachsenen Alter beginnen.

\section{Ätiologie}

Bereits beim Auftreten eines erstmaligen epileptischen Anfalls sollte der behandelnde Arzt eine ätiologische Klärung 
der Epilepsie anstreben. Eine Reihe von ätiologischen Gruppen sind geschaffen worden. Dabei lag der Schwerpunkt auf behandlungsrelevanten Ursachen. Häufig gehört eine zerebrale Bildgebung zu den zunächst veranlassten Untersuchungen, idealerweise eine MRT. Dies ermöglicht dem behandelnden Arzt zu entscheiden, ob eine strukturelle Läsion Ursache der vorliegenden Epilepsie sein könnte. Die 5 weiteren ätiologischen Gruppen sind: genetisch, infektiös, metabolisch und immunvermittelt sowie schließlich eine Gruppe für unbekannte Ätiologie (• Abb. 1). Die Epilepsie des Patienten kann dabei mehr als einer ätiologischen Kategorie zugeordnet werden, da diese untereinander keine hierarchische Beziehung aufweisen und weil die Bedeutung der ätiologischen Klassifikation kontextabhängig sein kann. Für einen Patienten mit tuberöser Sklerose besteht z. B. sowohl eine strukturelle als auch eine genetische Ätiologie. Die strukturelle Ätiologie ist für epilepsiechirurgische Therapieansätze von zentraler Bedeutung, die genetische Ätiologie hingegen Grundlage für humangenetische Beratungen sowie für neuartige Therapieformen, wie z.B. $\operatorname{mTOR}($ „mechanistic target of rapamycin“)-Inhibitoren.

\section{Strukturelle Ätiologie}

Das strukturellen Ätiologien zugrunde liegende Konzept ist, dass strukturelle Auffälligkeiten mit einem signifikant erhöhten Epilepsierisiko assoziiert sind, wie entsprechend konzipierte Studien nachweisen konnten [9]. Eine strukturelle Ätiologie bezeichnet Auffälligkeiten in der strukturellen Bildgebung, wobei die elektroklinische Beurteilung zusammen mit den Befunden der Bildgebung die Schlussfolgerung nahelegt, dass die strukturelle Läsion die wahrscheinliche Ursache der Anfälle des Patienten darstellt. Strukturelle Ätiologien können erworben sein, z.B. nach Schlaganfall, Trauma oder Infektion, oder genetisch bedingt sein, z.B. viele Fehlbildungen der kortikalen Entwicklung. Obwohl solche Fehlbildungen genetische Ursachen haben, wird die Epilepsie durch das strukturelle Korrelat bedingt. Der Nachweis einer möglicherweise sehr subtilen strukturellen Läsion erfolgt mittels MRTBildgebung unter Anwendung von epilepsiespezifischen Messprotokollen [37].

Es gibt allgemein anerkannte Assoziationen zwischen Epilepsien und strukturellen Läsionen, wie z.B. das relativ häufige Auftreten von Anfällen aus dem mesialen Temporallappen bei Hippocampussklerose. Andere wichtige Assoziationen sind u.a. die von gelastischen Anfällen bei hypothalamischen Hamartomen, das Rasmussen-Syndrom sowie das Hemikonvulsion-HemiplegieEpilepsie-Syndrom. Es ist wichtig, diese Assoziationen zu berücksichtigen, damit Bildgebungsdaten sorgfältig im Hinblick auf spezifische strukturelle Pathologien durchgesehen werden. Dies wiederum unterstreicht auch die Notwendigkeit einer epilepsiechirurgischen Diagnostik, falls medikamentöse Therapien erfolglos bleiben sollten.

Eine strukturelle Auffälligkeit kann genetisch bedingt oder erworben sein oder auch beides. Polymikrogyrien können beispielsweise die Folge von Mutationen in Genen wie dem GPR56 sein oder aber auch als erworbene Form nach intrauterinen Zytomegalievirusinfektionen auftreten [38]. Erworbene strukturelle Ursachen liegen $z$. B. bei hypoxischen Enzephalopathien sowie nach Traumata, Infektionen und Schlaganfällen vor. Es können beide ätiologische Kategorien, strukturell und genetisch, verwendet werden, wenn eine strukturelle Ätiologie eine bekannte genetische Grundlage aufweist, wie z. B. bei der tuberösen Sklerose, die durch eine Mutation in den Genen TSC1 oder TSC2 hervorgerufen wird. TSC1 kodiert dabei Hamartin und TSC2 Tuberin.

\section{Genetische Ätiologie}

Das Konzept einer genetischen Epilepsie ist es, dass eine Erkrankung, deren Kernsymptome epileptische Anfälle einschließen, direkt aus einer bekannten oder vermuteten genetischen Mutation heraus resultiert. Epilepsien, bei denen eine genetische Ätiologie diskutiert wird, sind relativ unterschiedlich, und in den meisten Fällen sind die betroffenen Gene bisher noch nicht bekannt.

Zunächst kann der Nachweis einer genetischen Ätiologie einzig auf der
Familienanamnese mit Hinweisen auf einen autosomal-dominanten Erbgang basieren. Bei dem Syndrom der "gutartigen familiären Neugeborenenkrämpfe “ weisen die meisten Familien Mutationen in einem der Kaliumkanalgene KCNQ2 oder KCNQ3 auf [39]. Im Fall des Syndroms der autosomal-dominanten nächtlichen Frontallappenepilepsie dagegen ist die zugrunde liegende Mutation derzeit lediglich bei wenigen betroffenen Individuen identifiziert worden [40]. Allerdings können auch klinische Studien an Gruppen von Patienten mit gleichen Syndromen, wie z.B. der kindlichen Absenceepilepsie oder der juvenilen myoklonischen Epilepsie, eine genetische Ätiologie nahelegen. Hinweise auf deren genetische Grundlage wurden in eleganten Studien wie der Zwillingsstudie von Lennox aus den 1950ern und von Familienaggregationsstudien gefunden [41, 42].

Als weitere Möglichkeit könnte eine molekulare Ursache identifiziert worden sein, die ein einzelnes erkranktes Gen oder eine Kopiezahlvariation mit signifikanter Bedeutung nahelegt. Bei einer zunehmenden Anzahl von Patienten werden genetische Abweichungen detektiert, die schwere und leichte Epilepsien verursachen können. Bei 30-50\% der Kleinkinder mit schweren „entwicklungsbedingten und epileptischen Enzephalopathien" können molekulargenetische Untersuchungen dafür verantwortliche $\mathrm{Mu}$ tationen in einer Vielzahl von Epilepsiegenen aufzeigen, wobei diese am häufigsten de novo auftraten [32]. Das bekannteste Beispiel ist das Dravet-Syndrom, bei dem über $80 \%$ der Patienten eine pathogene Variante von SCN1A vorweisen. Es ist dabei bemerkenswert, dass dieselbe monogene Ätiologie ein Spektrum von leichten bis schweren Epilepsien verursachen kann, wie z. B. die SCN1A-Mutationen. Sie kann das Dravet-Syndrom und generalisierte Epilepsie mit Fieberkrämpfen plus (GEFS+) verursachen, was unterschiedliche Behandlungspfade impliziert $[43,44]$.

Das Wissen um die phänotypische Varianz, die mit Mutationen in einem einzigen Gen einhergeht, ist wichtig, weil die Identifizierung einer Mutation in einem spezifischen Gen an sich meist nicht 
ausreicht, um deren Auswirkungen zu prognostizieren. Erst das elektroklinische Bild erlaubt die Beurteilung der klinischen Relevanz der jeweiligen Mutation. Dementsprechend erweist sich bisher der Großteil der Gene als phänotypisch heterogen und der Großteil der Syndrome als genetisch heterogen.

Wenn eine Epilepsie einer komplexen Vererbung folgt, die auf mehrere betroffene Gene mit oder ohne zusätzlich erforderliche Umwelteinflüsse schließen lässt, kann es doch möglich sein, Risikovarianten zu identifizieren, welche dann zur Entstehung der Epilepsie beitragen können. Diese sind aber alleine nicht hinreichend, um eine Epilepsie zu verursachen $[45,46]$. In solchen Fällen kann die Familienanamnese unergiebig sein, da andere Familienmitglieder oft nicht genügend viele der genetischen Varianten tragen, um von der Krankheit betroffen zu sein.

Es ist wichtig, sich vor Augen zu halten, dass "genetisch" nicht gleichbedeutend mit „vererbt“ ist. So werden bei schweren und leichten Epilepsien zunehmend De-novo-Mutationen nachgewiesen [47-52]. Dies bedeutet, dass die Mutation im betroffenen Patienten entstanden ist und es dementsprechend unwahrscheinlich ist, dass eine positive Familienanamnese in Bezug auf epileptische Anfälle vorhanden ist und dass die genetische Mutation nicht vererbt ist. Nichtsdestotrotz kann dieser Patient nun eine vererbbare Form der Epilepsie entwickelt haben. Wenn das Individuum beispielsweise eine dominante De-novo-Mutation in sich trägt, haben dessen Nachkommen ein $50 \%$ iges Risiko, die Mutation zu erben. Dies bedeutet jedoch nicht zwangsläufig, dass die Kinder unter Epilepsie leiden werden, da die Expression von der Penetranz der Mutation abhängt.

Noch tiefer gehend könnten Patienten auch Mosaikmutationen aufweisen. Dies bedeutet, dass 2 Zellpopulationen in demselben Patienten vorhanden sind, wobei eine Population die Mutation aufweist, während die andere das (normale) Wildtypallel in sich trägt. Bei Mosaikmutationen kann der Schweregrad einer Epilepsie variieren, d.h. niedrigere Mosaikraten resultieren in einer leichteren Form der Epilepsie, wie SCN1A-Studien gezeigt haben [53].
Eine genetische Ätiologie schließt Umwelteinflüsse als Kofaktoren nicht aus. Studien haben gezeigt, dass Umweltfaktoren zur Entstehung von epileptischen Anfällen beitragen können; beispielsweise können bei vielen Patienten mit Epilepsie Anfälle durch Schlafentzug, Stress oder andere Krankheiten provoziert werden. Eine genetische Ätiologie bedeutet somit, dass eine pathogene Variante (Mutation) signifikant an der Entstehung der individuellen Epilepsie beteiligt ist.

\section{Infektiöse Ätiologie}

Infektionen sind die weltweit häufigsten Ursachen von Epilepsie [54]. Eine Infektion als Ätiologie einer Epilepsie bedeutet, dass die Epilepsie die direkte Folge einer bekannten Infektion ist, wobei epileptische Anfälle ein Kernsymptom der Erkrankung darstellen. Eine infektiöse Ätiologie bezieht sich dabei auf Patienten mit Epilepsie und nicht auf Patienten, die Anfälle im Verlauf einer akuten Infektion erleiden, wie etwa bei Meningitis oder Enzephalitis. In bestimmten Regionen der Welt sind typische Beispiele dafür Neurozystizerkose, Tuberkulose, HIV, zerebrale Malaria, subakute sklerosierende Panenzephalitis, zerebrale Toxoplasmose und kongenitale Infektionen z. B. durch den Zika- oder Zytomegalievirus. Bei diesen Infektionen finden sich immer wieder strukturelle Korrelate. Eine infektiöse Ätiologie geht mit spezifischen Behandlungsoptionen einher. Infektionen können auch zu einer postinfektiösen Entwicklung einer Epilepsie führen, wenn z.B. eine virale Enzephalitis zu epileptischen Anfällen nach der akuten Infektion führt.

\section{Metabolische Ätiologie}

Eine Vielzahl metabolischer Erkrankungen ist mit Epilepsie assoziiert. Dieses Forschungsgebiet wächst, und ein besseres Verständnis der Bandbreite der verschiedenen Phänotypen entwickelt sich. Das Konzept einer metabolisch verursachten Epilepsie besteht darin, dass sie direkt aus einer bekannten oder angenommenen metabolischen Störung resultiert, bei der epileptische Anfälle ein Kernsymptom darstellen. Metabolische Ursachen beziehen sich auf einen umschriebenen Stoffwechseldefekt, der Symptome oder biochemische Veränderungen im gesamten Körper verursachen kann, wie Porphyrie, Urämie, Störungen des Aminosäurestoffwechsels oder Pyridoxin-abhängige Anfälle. In vielen Fällen gehen Stoffwechseldefekte mit einem genetischen Defekt einher. Wahrscheinlich haben die meisten metabolisch bedingten Epilepsien einen genetischen Hintergrund, wenn auch einige erworben sein können, wie z.B. der zerebrale Folsäuremangel. Die Identifizierung spezifischer metabolischer Ursachen von Epilepsien ist äußerst wichtig, da sich so Perspektiven zur Entwicklung spezifischer Therapien und möglicherweise zur Prävention geistiger Behinderung ergeben könnten.

\section{Immun vermittelte Ätiologie}

Das Konzept einer immun vermittelten Epilepsie besteht darin, dass die Erkrankung direkte Folge einer Störung des Immunsystems ist, bei der epileptische Anfälle als ein Kernsymptom auftreten. Eine Reihe immun vermittelter Epilepsien wurde kürzlich anhand charakteristischer Merkmale bei Erwachsenen wie auch bei Kindern beschrieben [54]. Eine immun vermittelte Ätiologie kann angenommen werden, wenn Hinweise für eine autoimmun vermittelte Entzündung des Zentralnervensystems bestehen. Die Diagnosen von Autoimmunenzephalitiden werden zunehmend häufiger. Die bessere Verfügbarkeit von entsprechenden Antikörperessays, z.B. bei AntiNMDA-Rezeptor Enzephalitis oder Anti-LGI1-Enzephalitis, hat daran einen großen Anteil [55]. Diese Fortschritte rechtfertigen eine eigene ätiologische Untergruppe, in der mit zielgerichteter Immunmodulation spezifische therapeutische Optionen existieren.

\section{Unbekannte Ätiologie}

„Unbekannt“ bedeutet, dass die Ursache der Epilepsie noch nicht bekannt ist. Dies trifft noch immer auf viele Patienten mit Epilepsie zu. Über die elektroklinische Einordnung z.B. als Frontallappenepilepsie hinaus, ist bei diesen Patienten eine weitere spezifischere Diagnose nicht möglich. Die Chance, eine Ursache der Epilepsie zu finden, hängt von der Ver- 


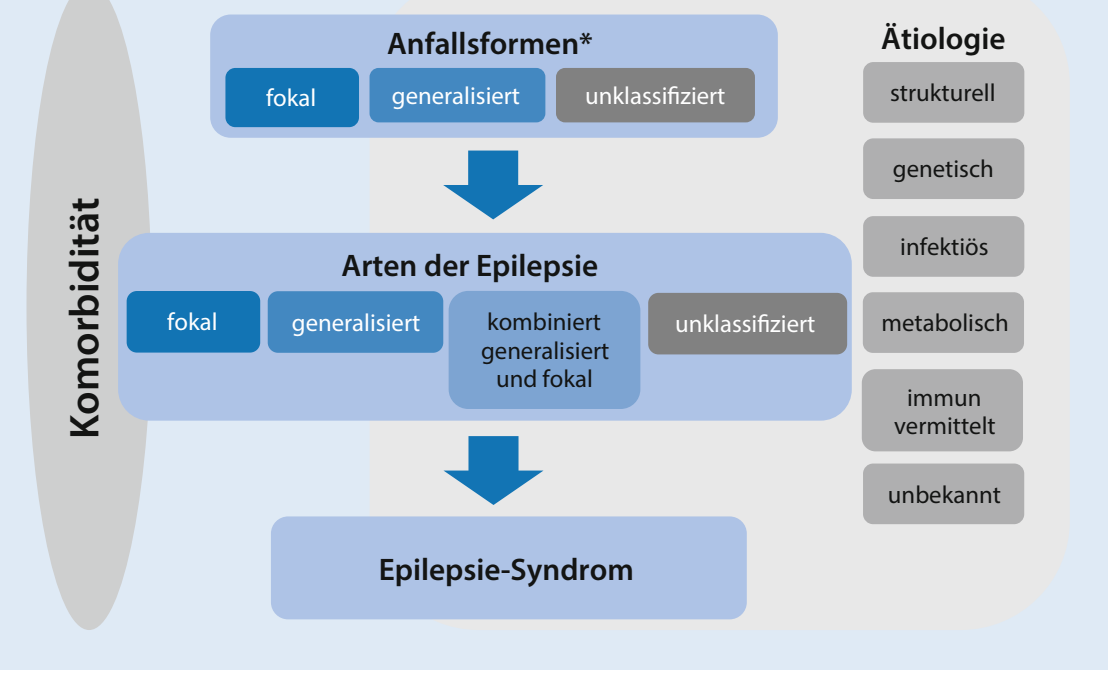

Abb. $1 \Delta$ Gerüst der Epilepsieklassifikation. $\left({ }^{*}\right.$ kennzeichnet Anfallsbeginn. Epilepsia ${ }^{\circledR}$ ILAE)

fügbarkeit von diagnostischen Möglichkeiten ab. Diese variieren je nach Gesundheitssystem und Land und werden sich hoffentlich mit der Zeit auch in Ländern mit limitierten Ressourcen verbessern.

\section{Komorbidität}

Es findet zunehmend Beachtung, dass viele Epilepsien mit Begleiterkrankungen wie Lernbehinderung, anderen neuropsychologischen Defiziten und Verhaltensauffälligkeiten einhergehen (• Abb. 1, vertikales Oval links). Diese können von unterschiedlicher Art und Schwere sein und erstrecken sich von leichten Lernschwierigkeiten hin zu geistiger Behinderung, von psychiatrischen Erkrankungen wie einer Autismus-Spektrum-Erkrankung und Depression bis hin zu psychosozialen Beschwerdebildern. Insbesondere bei schweren Epilepsien kann ein komplexes Zusammenspiel von Begleiterkrankungen beobachtet werden, das motorische Defizite wie Zerebralparese oder Verschlechterung des Gangbilds, Bewegungsstörungen, Skoliose, Schlafstörungen und gastrointestinale Beschwerden einschließt. Analog zur Ätiologie der Epilepsien sollten mögliche Begleiterkrankungen bei jedem Patienten mit Epilepsie auf jeder Stufe der Klassifikation mit einbezogen werden, sodass diese frühzeitig iden- tifiziert, diagnostiziert und behandelt werden können.

\section{Neue Terminologie und Definitionen}

\section{Entwicklungsbedingte und epileptische Enzephalopathie}

Der Begriff „epileptische Enzephalopathie" wurde von Berg et al. [9] neu gefasst. Sie ist als eine Enzephalopathie definiert, bei der durch epileptische Aktivität selber eine schwerere Beeinträchtigung von Kognition und Verhalten hervorgerufen wird als üblicherweise die zugrunde liegende Pathologie alleine verursachen würde (z. B. bei kortikalen Fehlbildungen). Diese diffusen oder spezifischen Beeinträchtigungen können mit der Zeit zunehmen. Derartige Defizite können bei allen Epilepsien in jedem Alter mit großer Spannbreite auftreten.

Das Konzept der epileptischen Enzephalopathie kann auf Epilepsien in jeder Altersstufe angewendet werden und sollte eine breitere Anwendung finden als nur bei schweren Epilepsien mit Beginn im Säuglingsalter oder in der Kindheit. Viele der mit einer Enzephalopathie assoziierten Epilepsiesyndrome sind genetisch bedingt. Beispiele sind das West-Syndrom, welches eine ausgeprägte genetische Heterogenität aufweist, und die epileptische Enzephalopathie mit kontinu- ierlichem Spike-und-Wave während des Schlafes (CSWS), für welche mittlerweile die ersten assoziierten Gene entdeckt worden sind [32]. Ebenso können Enzephalopathiesyndrome eine erworbene Ursache haben, wie z. B. die hypoxische Enzephalopathie oder Enzephalopathien als Folge von Schlaganfall oder bei kortikalen Fehlbildungen, die wiederum selbst eine genetische oder erworbene Ursache haben können.

Das Konzept einer epileptischen Enzephalopathie kann auch auf monogenetische Erkrankungen angewandt werden, beispielsweise bei CDKL5-Enzephalopathie oder CHD2-Enzephalopathie. Ein und dieselbe Mutation kann bei einigen Patienten zu einer epileptischen Enzephalopathie führen und bei anderen Patienten lediglich eine selbstlimitierte Epilepsie hervorrufen. Beispiele hierfür sind Mutationen in den Genen SCN1A, SCN2A, SLC2A1, KCNQ2, KCNA2 und CHD2. Bei einer epileptischen Enzephalopathie beeinträchtigt häufige epilepsietypische Aktivität die geistige Entwicklung, woraus eine kognitive Verlangsamung und häufig auch eine Regression resultieren. Mitunter sind auch psychiatrische Komorbidität und Verhaltensauffälligkeiten die Folge. Epilepsietypische Aktivität kann sowohl bei Patienten mit bis dato normaler Entwicklung als auch bei solchen mit vorbestehender Entwicklungsverzögerung eine Regression verursachen. Bei Letzteren kann neben einer Regression auch zunächst eine stagnierende Entwicklung beobachtet werden. Ein zentraler Aspekt dieses Konzepts ist es, dass eine Reduktion epilepsietypischer Aktivität möglicherweise derartigen Entwicklungsverzögerungen entgegenwirken könnte. Dies ist aus klinischer Sicht ein wichtiger Gesichtspunkt, der häufig durch die Familien und die Behandler bestätigt wird.

Bei vielen der schwer verlaufenden, genetischen Erkrankungen finden sich neben den Entwicklungsverzögerungen, die durch häufige epilepsietypische Aktivität verursacht werden, auch solche, die direkt auf die Genmutation zurückzuführen sind.

Dieses komplexe Zusammenspiel kann sich verschiedenartig manifestieren. So kann z. B. bei einer vorbeste- 
henden Entwicklungsverzögerung eine Entwicklungsstagnation oder sogar eine Regression eintreten, wenn die Anfälle beginnen oder prolongiert auftreten. Bei anderen Formen kann eine Entwicklungsverzögerung vor dem Hintergrund einer bislang normalen Entwicklung einsetzen, auch bevor epilepsietypische Potenziale im EEG auftreten. Ein bekanntes Beispiel dafür ist die häufige Enzephalopathie beim Dravet-Syndrom. Eine verlangsamte Entwicklung oder sogar eine Regression tritt im Alter von 1 oder 2 Jahren auf, auch wenn zu dieser Zeit epilepsietypische Aktivität im EEG normalerweise noch nicht häufig $\mathrm{zu}$ sehen ist. Dies weist auf einen entwicklungsbedingten Aspekt der Erkrankung zusätzlich zur Epilepsie hin. Beide Arten der Beeinträchtigung sind die Folge einer zugrunde liegenden Mutation im Natriumkanalgen SCN1A, die in $>80 \%$ der Fälle gefunden wird. In einer dritten Gruppe kann die Epilepsie relativ früh in der kindlichen Entwicklung wieder aufhören, aber deren negative Konsequenzen für die Entwicklung können weiter deutlich $\mathrm{zu}$ sehen sein wie bei einigen Patienten mit KCNQ2-Enzephalopathie oder STXBP1-Enzephalopathie. Diese Beobachtungen lassen sich aufviele genetische Enzephalopathien übertragen und legen eine Erweiterung der Terminologie nahe. Wo zutreffend, sollte also der Begriff ,entwicklungsbedingt“ in die Krankheitsbezeichnung aufgenommen werden. Dies unterstreicht, dass beide Aspekte eine Rolle in der klinischen Manifestation des Syndroms spielen. Diese Konzepte sind für Angehörige und den behandelnden Arzt von zentraler Bedeutung, um den Krankheitsverlauf zu verstehen.

Dementsprechend wird vorgeschlagen, dass die Bezeichnung „entwicklungsbedingte und epileptische Enzephalopathie“, wo zutreffend, auf Patienten jeden Alters angewendet wird. Die Verwendung von einem oder beiden Deskriptoren ist zulässig; „entwicklungsbedingte Enzephalopathie“, wenn die Beeinträchtigung der Entwicklung des Patienten ohne schwere Epilepsie im Vordergrund steht, wobei Regression oder weitere Verlangsamung der Entwicklung vergesellschaftet sein kann; „epilepti- sche Enzephalopathie“ bei denjenigen Fällen, bei denen keine vorbestehende Entwicklungsverzögerung vorhanden ist und die genetische Mutation als solche bekanntermaßen ohne Entwicklungsverzögerung einhergeht; und schließlich „entwicklungsbedingte und epileptische Enzephalopathie“, wenn beide Faktoren für die klinische Manifestation eine Rolle spielen. Häufig wird es nicht möglich sein festzulegen, ob die epileptische oder entwicklungsbedingte Komponente der Erkrankung einen größeren Einfluss auf das klinische Erscheinungsbild hat.

Bislang wurden viele Patienten mit diesen Erkrankungen als „symptomatisch generalisierte Epilepsien“ klassifiziert; diese Bezeichnung sollte jedoch nicht mehr verwendet werden, da sie bei einer sehr heterogenen Patientengruppe Anwendung fand. Sie wurde auf Patienten mit entwicklungsbedingten Enzephalopathien und Epilepsie (z.B. mit infantiler Zerebralparese, geistiger Behinderung und leichter Epilepsie) angewendet, aber auch bei Patienten mit epileptischer Enzephalopathie oder auch entwicklungsbedingter und epileptischer Enzephalopathie benutzt. Selbst bei einigen Patienten mit generalisierter Epilepsie oder kombinierter generalisierter und fokaler Epilepsie wurde sie verwendet. Die neue Klassifikation ermöglicht eine genauere Klassifizierung der Epilepsien dieser Patienten.

In vielen Fällen, in denen eine genetische Mutation von klinisch relevanter Wirkung identifiziert wird, können die Begriffe „entwicklungsbedingte und epileptische Enzephalopathie" unter dem Namen des zugrunde liegenden Syndroms subsumiert werden. So können viele der bekannten entwicklungsbedingten und epileptischen Enzephalopathien nach dem zugehörigen Gen in Verbindung mit dem Wort „Enzephalopathie“ benannt werden; Beispiele sind „STXBP1-Enzephalopathie“ oder „CNQ2-Enzephalopathie“. Dies ist besonders dann wichtig, wenn man sich auf eine genetische Krankheit bezieht, bei der bestimmte Gene sowohl mit schweren als auch mit selbstlimitierten, pharmakoresponsiven Epilepsien assoziiert sein können, wie z. B. bei dem KCNQ2oder SCN2A-Gen. Hier kann der Begriff
„Enzephalopathie“ benutzt werden, um die schwere Form der Krankheit mit Entwicklungsverzögerung zu benennen.

\section{Selbstlimitiert und pharmako- responsiv}

Je mehr Wissen über die Auswirkungen von Komorbidität auf die Lebensqualität der Betroffenen akkumuliert wurde, desto größer wurden Bedenken, dass der Begriff „,benigne“ diesem Sachverhalt nicht gerecht wird. Dies gilt insbesondere für leichtere Epilepsiesyndrome wie die „benigne Epilepsie mit zentrotemporalen Spikes“ (BECTS) und die „kindliche Absenceepilepsie“ („childhood absence epilepsy“ [CAE]). Trotz des Eindrucks eines benignen Epilepsiesyndroms kann BECTS mit transienten oder sogar permanenten kognitiven Beeinträchtigungen verbunden sein $[56,57]$, und CAE ist mit einem erhöhten Risiko für negative psychosoziale Folgen assoziiert wie einer frühen, ungewollten Schwangerschaft [58].

Der Bericht von Berg et al. [9] schlug daher neue Begrifflichkeiten vor, um die eigentliche Intention des Begriffs „benigne“ deutlicher herauszustellen. Deswegen wird „benigne“ als Deskriptor für Epilepsie durch „selbstlimitiert“ und „pharmakoresponsiv“ ersetzt. Beide Begriffe ersetzen verschiedene Elemente der Bedeutung von „benigne“. „Selbstlimitiert" bezieht sich auf die wahrscheinlich spontane Ausheilung des Syndroms und "pharmakoresponsiv“ weist darauf hin, dass ein Epilepsiesyndrom durch eine adäquate antiepileptische Therapie kontrollierbar ist. Dabei muss allerdings berücksichtig werden, dass Patienten mit diesen Syndromen auch nicht pharmakoresponsiv sein können. Wie oben bereits erwähnt, existiert keine formelle ILAE-Klassifikation von Syndromen; jedoch steht zu erwarten, dass der Begriff „benigne“ in der Bezeichnung der jeweiligen Syndrome im Laufe der Zeit ersetzt wird. Begriffe wie „maligne“ und „katastrophal“" werden ebenfalls nicht mehr benutzt. Sie werden aufgrund ihrer ernsten und verheerenden Konnotationen aus dem epileptologischen Wortschatz entfernt. 
Es besteht die Hoffnung, dass diese neue Klassifikation der Epilepsien Behandlern wie Betroffenen und ihren Angehörigen einen guten Dienst erweisen wird und zu verbesserten Diagnosen, einem Verständnis der Ätiologie und zu gezielten Therapien der individuellen Krankheiten führen wird. Allerdings bleibt es weiter Aufgabe, die zugrunde liegenden Pathomechanismen wiederkehrender epileptischer Anfälle zu erforschen, auch wenn die Ätiologie klar definiert ist.

Erhebliche Erkenntnisfortschritte in der Neurobiologie von Anfällen und Epilepsien führten $\mathrm{zu}$ einem grundlegenden Paradigmenwechsel in den Konzepten, auf denen die Klassifikation der Epilepsien basiert. Die vorliegende Klassifikation wurde daher notwendig, um den aktuellen Kenntnisstand widerzuspiegeln. Sie soll für Forschung und Klinik ein überaus nützliches Kommunikationsmittel sein.

\section{Korrespondierender Übersetzer}

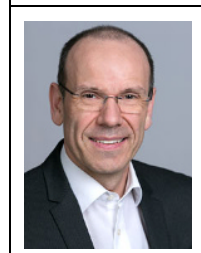

Prof. Dr. Hajo Hamer, MHBA

Klinik für Neurologie

Universitätsklinikum Erlangen

Schwabachanlage 6

91054 Erlangen

hajo.hamer@uk-erlangen.de

\section{Korrespondenzadresse}

\section{Ingrid E. Scheffer}

Epilepsy Research Centre

245 Burgundy St, 3084 Heidelberg, Victoria,

Australien

scheffer@unimelb.edu.au

\section{Einhaltung ethischer Richtlinien}

Interessenkonflikt. I.E. Scheffer erhielt Unterstützung und/oder Beratungshonorare von UCB, Eisai, Athena Diagnostics, GlaxoSmithKline, Transgenomics und Biocodex. Sie ist Mitglied der Herausgeberschaft von Neurology und Epileptic Disorders. Sie erhielt Fördermittel von NHMRC, ARC, NIH, HRC, CURE, US Department of Defense und March of Dimes. S. Ber- kovic erhielt Zahlungen von UCB Pharma, Novartis Pharmaceuticals, Sanofi-Aventis, und Jansen Cilag fü Beratungen und Fortbildungen und hält ein Patent für eine SCN1A-Testung, die von Bionomics Inc für ver schiedene Diagnostikunternehmen lizensiert wurde. G. Capovilla ist Mitglied der Herausgeberschaft des European Journal of Pediatric Neurology. M.B. Connolly hat Forschungsförderung und/oder Referentenhonorare erhalten von UCB, Novartis, Biocodex, Eisai und Sage Therapeutics. Die gesamten Honorare wurden der Epilepsy Research and Development Fund gespen det. Sie erhielt auch Forschungsförderung von CIHR (Canadian Institute for Health Research) und The Alva Foundation. Sie ist Kovorsitzende des Canadian Paediatric Epilepsy Network. J. French Das Epilepsy Study Consortium kompensiert ihre Universität für Beratungen von Acorda, Anavex, Brabant Pharma, BioPharm Solutions, Eisai Medical Research, GlaxoSmithKline, GW Pharma, Impax, Johnson and Johnson, Marinus, Neusentis, Novartis, Roivant, Pfizer, Sage, Sunovion, SK life sciences, Supernus Pharmaceuticals, Takeda, UCB, Upsher-Smith, Ultragenyx, Vertex, Zogenix, Zynerba sowie für wissenschaftliche Beratung von Anavex, UCB. Forschungsförderung durch Acorda, Alexza, LCGH, Eisai Medical Research, Lundbeck, Pfizer, SK life sciences, UCB, Upsher-Smith, Vertex und durch NINDS, Epilepsy Therapy Project, Epilepsy Research Foundation, Epilepsy Study Consortium. Sie ist Mitglied der Herausgeberschaft von Lancet Neurology, Neurology Today und Epileptic Disorders und ist bezahlte Mitherausgeberin von Epilepsia. L. Guilhoto ist Mitglied der Herausgeberschaft von Seizure - European Journal of Epilepsy. E. Hirsch erhielt Unterstützung von UCB und/oder Beratungshonorare von UCB, ESAI, BIAL. G.W. Mathern erhält in Teilen seiner beruflichen Tätigkeit Unterstützung durch Davies/Crandall Chair for Epilepsy Research der UCLA und ist Mitherausgeber von Epilepsia und Epilepsia Open. Er ist auch Mitglied der Herausgeberschaft von Neurology und Mitglied des Data Management Committee von NeuroPace, Inc. S.L. Moshé MD ist Vorsitzender des Charles Frost Chair in Neurosurgery and Neurology und erhält Fördermittel von NIH NS43209 und 1U54NS100064-01, CURE Infantile Spasms Initiative, US Department of Defense (W81XWH-13-1-0180), der Heffer Family und der Segal Family Foundations sowie von den Abbe Goldstein/Joshua Lurie und Laurie Marsh/Dan Levitz Familie. Er ist Mitherausgeber von Neurobiology of Disease und ist Mitglied der Herausgeberschaft von Brain and Development, Pediatric Neurology und Physiolo gical Research. Er halt eine jährliche Kompensation von Elsevier für seine Tätigkeit als Mitherausgeber von Neurobiology of Disease und Tantiemen von 2 Büchern, die er mit herausgab. Er erhielt Beratungshonorare von Eisai und UCB. D.R. Nordli erhält Fördermittel von NIH (1-RO1-NS43209) und CURE. Er ist Mitherausgeber von UpToDate. E. Perucca erhielt Referentenoder Beratungshonorare oder Forschungsförderung von Eisai, Biopharm Solutions, GW Pharma, Mylan, Sanofi, SK life sciences, Sun Pharma, Takeda und UCB Pharma. T. Tomson erhielt Forschungsförderung und/ oder Referentenhonorare an seinen Arbeitgeber von Eisai, GlaxoSmithKline, Novartis, Bial, und UCB. Er erhielt auch Forschungsförderung von CURE, Stockholm County Council und der EU (DG Sante). S. Wiebe erhielt Referenten- oder Beratungshonorare und/ oder Fördermittel für Forschung bzw. Fortbildung von UCB, Electrocore und Sunovion. S.M. Zuberi erhielt Forschungsförderung und/oder Referenten-/ Beratungshonorare von Epilepsy Research UK, Dravet Syndrome UK, UCB Pharma, Yorkhill Children's Charity, GW Pharma, Brabant Pharma and Zogenix. Er ist Herausgeber von European Journal of Paediatric
Neurology. S. Jain und Y.-H. Zhang geben an, dass kein Interessenkonflikt besteht.

Dieser Beitrag beinhaltet keine von den Autoren durchgeführten Studien an Menschen oder Tieren.

\section{Literatur}

1. Gastaut H, Caveness WF, Landolt W et al (1964) A proposed international classification of epileptic seizures. Epilepsia 5:297-306

2. Gastaut H (1969) Clinical and electroencephalographical classification of epileptic seizures. Epilepsia 10(Suppl):2-13

3. Gastaut H (1969) Classification of the epilepsies. Proposal for an international classification. Epilepsia 10(Suppl):14-21

4. Commission on Classification and Terminology of the International League Against Epilepsy (1985) Proposal for classification of epilepsies and epileptic syndromes. Epilepsia 26:268-278

5. Commission on Classification and Terminology of the International League Against Epilepsy (1989) Proposal for revised classification of epilepsies and epileptic syndromes. Epilepsia 30:389-399

6. Commission on Classification and Terminology of the International League Against Epilepsy (1981) Proposal for revised clinical and electroencephalographic classification of epileptic seizures. Epilepsia 22:489-501

7. Engel J Jr. (2001) A proposed diagnostic scheme for people with epileptic seizures and with epilepsy: report of the ILAE Task Force on Classification and Terminology. Epilepsia 42:796-803

8. Engel J (2006) Report of the ILAE classification core group. Epilepsia 47:1558-1568

9. Berg AT, Berkovic SF, Brodie MJ et al (2010) Revised terminology and concepts for organization of seizures and epilepsies: report of the ILAE Commission on Classification and Terminology, 2005-2009. Epilepsia 51:676-685

10. Fisher RS, Cross JH, French JA et al (2017) Operational classification of seizure types by the International League Against Epilepsy. Epilepsia. https://doi.org/10.1111/epi.13671

11. Epilepsy ILa (2014) Guidelines for publications from league commissions and task forces. http://www.ilae.org/visitors/centre/guidelines. cfm. Zugegriffen: 2. Juli 2015

12. Avanzini G (2010) A sound conceptual framework for an epilepsy classification is still lacking Epilepsia 51:720-722

13. Beghi E (2011) New classification proposals for epilepsy: a real advancement in the nosography of the disease? Epilepsia 52:1197-1198 (discussion 1205-1199)

14. Berg AT, Scheffer IE (2011) New concepts in classification of the epilepsies: entering the 21st century. Epilepsia 52:1058-1062

15. Berg AT, Scheffer IE (2011) What is at stake in a classification? Epilepsia 52:1205-1208

16. Camfield $P(2012)$ Issues in epilepsy classification forpopulation studies. Epilepsia 53(Suppl2):10-13

17. Duncan JS (2011) The evolving classification of seizures and epilepsies. Epilepsia 52:1204-1205 (discussion 1205-1209)

18. Engel J Jr (2011) The etiologic classification of epilepsy. Epilepsia 52:1195-1197 (discussion 1205-1209)

19. Ferrie CD (2010) Terminology and organization of seizures and epilepsies: radical changes not justified by new evidence. Epilepsia 51:713-714 
20. Fisher RS (2010) What is a classification essay? Epilepsia 51:714-715

21. Guerrini R (2010) Classification concepts and terminology: is clinical description assertive and laboratory testing objective? Epilepsia 51:718-720

22. Jackson G (2011) Classification of the epilepsies 2011. Epilepsia 52:1203-1204 (discussion 1205-1209)

23. MosheSL (2011) In support of the ILAECommission classification proposal. Epilepsia 52:1200-1201 (discussion 1205-1209)

24. Luders HO, Amina S, Baumgartner C et al (2012) Modern technology calls for a modern approach to classification of epileptic seizures and the epilepsies. Epilepsia 53:405-411

25. Panayiotopoulos CP (2012) The new ILAE report on terminology and concepts for the organization of epilepsies: critical review and contribution. Epilepsia 53:399-404

26. Shinnar S (2010) The new ILAE classification. Epilepsia 51:715-717

27. Shorvon SD (2011) The etiologic classification of epilepsy. Epilepsia 52:1052-1057

28. Wolf $P$ (2010) Much ado about nothing? Epilepsia 51:717-718

29. Wong M (2011) Epilepsy is both a symptom and a disease: a proposal for a two-tiered classification system. Epilepsia 52:1201-1203 (discussion 1205-1209)

30. Schefferl, French J, Hirsch Eetal (2016) Clasification of the epilepsies: New concepts for discussion and debate-Special report of the ILAE Classification Task Force of the Commission for Classification and Terminology. Epilepsia Open 1:37-44

31. Fisher RS, Acevedo C, Arzimanoglou A et al (2014) ILAE official report: a practical clinical definition of epilepsy. Epilepsia 55:475-482

32. McTague A, Howell KB, Cross JH et al (2016) The genetic landscape of the epileptic encephalopathies of infancy and childhood. Lancet Neurol 15:304-316

33. Guerrini R, Pellacani S (2012) Benign childhood focal epilepsies. Epilepsia 53(Suppl 4):9-18

34. Beaumanoir A, Nahory A (1983) Benign partial epilepsies: 11 cases of frontal partial epilepsy with favorable prognosis. Rev Electroencephalogr Neurophysiol Clin 13:207-211

35. Berkovic SF, Mclntosh A, Howell RA et al (1996) Familial temporal lobe epilepsy: a common disorder identified in twins. Ann Neurol 40:227-235

36. Sveinbjornsdottir S, Duncan JS (1993) Parietal and occipital lobe epilepsy: a review. Epilepsia 34:493-521

37. Gaillard WD, Chiron C, Cross JH et al (2009) Guidelines for imaging infants and children with recent-onset epilepsy. Epilepsia 50:2147-2153

38. Guerrini R, Dobyns WB (2014) Malformations of cortical development: clinical features and genetic causes. Lancet Neurol 13:710-726

39. Grinton BE, Heron SE, Pelekanos JT et al (2015) Familial neonatal seizures in 36 families: Clinical and genetic features correlate with outcome. Epilepsia 56:1071-1080

40. Tinuper P, Bisulli F, Cross JH et al (2016) Definition and diagnostic criteria of sleep-related hypermotor epilepsy. Neurology 86:1834-1842

41. Lennox WG (1947) The genetics of epilepsy. Am J Psychiatry 103:457-462

42. Lennox WG (1951) The heredity of epilepsy as told by relatives and twins. J Am Med Assoc 146:529-536

43. Brunklaus A, Dorris L, Ellis R et al (2013) The clinical utility of an SCN1A genetic diagnosis in infantileonset epilepsy. Dev Med Child Neurol 55:154-161
44. Mullen SA, Scheffer IE(2009) Translational research in epilepsy genetics: sodium channels in man to interneuronopathy in mouse. Arch Neurol 66:21-26

45. Helbig I, Mefford H, Sharp A et al (2009) 15q13. 3 microdeletions increase risk of idiopathic generalized epilepsy. Nat Genet 41:160-162

46. Dibbens LM, Mullen S, Helbig I et al (2009) Familial and sporadic 15q13. 3 microdeletions in idiopathic generalized epilepsy: precedent for disorders with complex inheritance. Hum Mol Genet 18:3626-3631

47. Claes L, Del-Favero J, Ceulemans B et al (2001) De novo mutations in the sodium-channel gene SCN1A cause severe myoclonic epilepsy of infancy. Am JHum Genet 68:1327-1332

48. Weckhuysen S, Mandelstam S, Suls A et al (2012) KCNQ2 encephalopathy: emerging phenotype of a neonatal epileptic encephalopathy. Ann Neurol 71:15-25

49. Depienne C, Bouteiller D, Keren B et al (2009) Sporadic infantile epileptic encephalopathy caused by mutations in PCDH19 resembles Drave syndrome but mainly affects females. Plos Genet 5:e1000381

50. Arsov T, Mullen SA, Rogers S et al (2012) Glucose transporter 1 deficiency in the idiopathic generalized epilepsies. Ann Neurol 72:807-815

51. Scheffer IE, Grinton BE, Heron SE et al (2012) PRRT2 phenotypic spectrum includes sporadic and fever-related infantile seizures. Neurology 79:2104-2108

52. Carvill GL, Heavin SB, Yendle SC et al (2013) Targeted resequencing in epileptic encephalopathies identifies de novo mutations in CHD2 and SYNGAP1. Nat Genet 45:825-830

53. Depienne C, Trouillard O, Gourfinkel-An I et al (2010) Mechanisms for variable expressivity of inherited SCN1A mutations causing Dravet syndrome. J Med Genet 47:404-410

54. Vezzani A, Fujinami RS, White HS et al (2016) Infections, inflammation and epilepsy. Acta Neuropathol 131:211-234

55. Lancaster E, Dalmau J (2012) Neuronal autoantigens-pathogenesis, associated disorders and antibody testing. Nat Rev Neurol 8:380-390

56. Staden UE, Isaacs E, Boyd SG et al (1998) Language dysfunction in children with rolandic epilepsy. Neuropediatrics 29:242-248

57. Lillywhite LM, Saling MM, Harvey AS et al (2009) Neuropsychological and functional MRI studies provide converging evidence of anterior language dysfunction in BECTS. Epilepsia 50:2276-2284

58. Wirrell EC, Camfield CS, Camfield PR et al (1997) Long-term psychosocial outcome in typical absence epilepsy. Sometimes a wolf in sheeps' clothing. Arch Pediatr Adolesc Med 151:152-158 